\title{
A Comparison of Azathioprine and Mycophenolate Mofetil as Adjuvant Drugs in Patients with Pemphigus: A Retrospective Cohort Study
}

\author{
Siriorn Sukanjanapong - Darin Thongtan • Silada Kanokrungsee • \\ Poonkiat Suchonwanit · Kumutnart Chanprapaph (D)
}

Received: October 24, 2019 / Published online: December 21, 2019

(C) The Author(s) 2019

\begin{abstract}
Introduction: Azathioprine (AZA) and mycophenolate mofetil (MMF) are both first-line steroid-sparing agents used for the treatment of pemphigus in combination with a corticosteroid, but few studies to date have directly compared these two combination treatment modalities. The aim of this study was to compare the efficacy and safety of each of these agents as adjuvant therapy with the corticosteroid prednisolone for the treatment of pemphigus, using standardized outcome parameters.

Methods: This was a retrospective study of patients with pemphigus who received corticosteroid therapy in combination with either AZA or MMF at the Autoimmune Blistering Skin Diseases Clinic of Ramathibodi Hospital (Bangkok) between January 2007 and July 2017. The
\end{abstract}

Enhanced Digital Features To view enhanced digital features for this article go to https://doi.org/10.6084/ m9.figshare.11298938.

S. Sukanjanapong $\cdot$ D. Thongtan · P. Suchonwanit . K. Chanprapaph ( $\square)$

Division of Dermatology, Department of Medicine, Faculty of Medicine, Ramathibodi

Hospital-Mahidol University, Bangkok, Thailand

e-mail: kumutnartp@hotmail.com

S. Kanokrungsee

Skin Center, Srinakharinwirot University, Wattana

District, Bangkok, Thailand treatment response was evaluated using early [end of the consolidation phase (ECP)] and late endpoints [complete remission (CR) on therapy, CR off therapy and immunological remission]. Cumulative steroid use, relapse rate and adverse events in each treatment group were also compared.

Results: Of the 62 patients with pemphigus included in the study, 37 were treated with prednisolone plus AZA as adjuvant (AZA group) and 25 patients were treated with prednisolone plus MMF as adjuvant (MMF group). The majority of patients in both treatment groups reached the ECP (AZA group 88.2\%; MMF group $71.4 \%$; between-group difference not statistically significant at $p=0.156$ ); the median time required to achieve this early endpoint was also comparable $(p=0.362)$. A high percentage of patients in both the AZA and MMF groups attained CR on therapy (AZA 73\%; MMF 72\%). The total number of patients who achieved CR on and off therapy were comparable in the two groups ( $p=0.933$ and $p=0.690$, respectively). However, the median time for patients to achieve CR on therapy was significantly shorter for those on MMF than for those on AZA (7.3 vs. 12.5 months; $p=0.019$ ), and the cumulative steroid dose required for patients to achieve CR both on and off therapy was significantly lower in the MMF group than in the AZA group ( $p=0.007$ and $p=0.043$, respectively).

Conclusion: While corticosteroid in combination with either AZA or MMF is an effective 
therapeutic regimen for the treatment of pemphigus, MMF demonstrates a shorter time to achieve CR on therapy and has a significantly higher steroid-sparing effect.

Keywords: Adjuvant drug therapy; Azathioprine; Corticosteroid; Mycophenolate mofetil; Pemphigus

\section{Key Summary Points}

Azathioprine (AZA) and mycophenolate mofetil (MMF) are first-line steroidsparing agents used in combination with a corticosteroid for the treatment of pemphigus, but few direct comparisons between these two treatment modalities have been made.

Reliable data on whether AZA or MMF differ in efficacy may be valuable, especially in financially restrained countries where anti-CD20 antibodies are not readily available or affordable.

Corticosteroid in combination with either AZA or MMF is an effective treatment regimen for pemphigus, with similarly high rates of patients on AZA or MMF as adjuvant achieving remission.

Compared to AZA, MMF demonstrates a shorter time to achieve complete remission in patients with pemphigus on therapy and a significantly higher steroidsparing effect.

\section{INTRODUCTION}

Pemphigus is a group of potentially life-threatening autoimmune mucocutaneous disorders with an incidence of 1-16 new cases per million per year $[1,2]$. Autoantibodies targeting different proteins of demosomes, particularly antidesmoglein (anti-dsg 1) and anti-desmoglein 3 (anti-dsg 3), lead to chronic and severe intraepidermal blisters. Systemic corticosteroids have long been the mainstay treatment modality, resulting in reductions in the mortality rate from $>70 \%$ to $<10 \%$ [3]. However, the side effects from prolonged corticosteroid use have driven the search for the best steroid-sparing agent among currently available immunosuppressants, including azathioprine (AZA), mycophenolate mofetil (MMF), cyclophosphamide, cyclosporine, among others [4-6].

Due to the rarity of the disease, therapeutic prospective controlled trials are limited and management by professionals varies considerably [7]. A majority of guidelines for pemphigus treatment are derived from expert opinions [8-12]. In the most recent international consensus, rituximab was added as first-line treatment alongside corticosteroids, and AZA and MMF were listed as first-line steroid-sparing agents [12]. The consensus also provides definitions for disease outcome parameters [13], which permit a more accurate evaluation of drug efficacy in clinical studies.

AZA is a synthetic purine analog that is metabolized in the liver to 6-mercaptopurine and its active metabolite 6-thioguanine. This metabolite interferes with DNA replication and suppresses T-cell functions and B-cell antibody production [14]. The therapeutic combination of AZA + corticosteroid for the treatment of pemphigus is recognized as being both effective and safe $[15,16]$. A meta-analysis has demonstrated that using AZA as adjuvant therapy with systemic corticosteroids has a steroid-sparing effect when compared to corticosteroid treatment alone [5]. MMF is the prodrug of mycophenolic acid, an active metabolite that can selectively inhibit the de novo purine synthesis specific to lymphocytes [17]; consequently, it is considered to be a more specific treatment than AZA. MMF was introduced into clinical use subsequent to AZA as effective adjuvant treatment for pemphigus and shown to be able to induce remission in patients with pemphigus who relapsed after AZA + corticosteroid combination therapy [18, 19], even in resistant cases [20]. The results of a multicenter randomized control trial that compared the combination MMF + corticosteroid to corticosteroid therapy alone led the authors to conclude that while there were no differences in the 
percentages of patients achieving absence of lesions, the group treated with the MMF + cortcosteroid combination showed faster and more durable responses than the prednisoloneonly group [21]. This result conflicts with that reported in another study involving newly diagnosed patients, with the authors reporting no superiority of MMF + cortcosteroid combination therapy in terms of response to treatment or prevention of relapses over corticosteroid monotherapy [22]. Although AZA and MMF are widely accepted immunosuppressants, only a few studies have directly compared these two drugs, and these studies were conducted before the publication of standardized endpoints [23, 24].

Reliable data on whether AZA or MMF differ in efficacy may be valuable, especially in financially restrained countries where rituximab is not readily available or affordable and combination treatment with corticosteroids and adjuvant immunosuppressants are the only option. Thus, the aim of our study was to compare the efficacy and safety of each of these agents as adjuvant therapy with the corticosteroid prednisolone (AZA + prednisolone vs. MMF + prednisolone) for the treatment of pemphigus, using the recommended outcome parameters.

\section{METHODS}

This retrospective study was approved by the Mahidol University Institutional Review Board (ID 08-60-74) and conducted at the Autoimmune Blistering Skin Diseases Clinic, Division of Dermatology, Ramathibodi Hospital, Bangkok, Thailand. All procedures performed in studies involving human participants were in accordance with the institutional research committee and with the 1964 Helsinki Declaration and its later amendments or comparable ethical standards. Informed consent was obtained from all individual participants included in the study.

Medical records of patients with pemphigus treated at our clinic between January 2007 and July 2017 were retrieved and analyzed. Inclusion criteria were: age $>15$ years; diagnosis of pemphigus vulgaris (PV) or pemphigus foliaceus (PF) based on the typical clinical presentations, histopathology, immunofluorescence studies and enzyme-linked immunosorbent assays (ELISA) for the anti-desmoglein autoantibodies anti-dsg1 and anti-dsg3; and treatment with prednisolone in combination with either AZA or MMF as mono-adjuvant therapy. Exclusion criteria were: coexisting autoimmune diseases; multiple drug use; inconsistency with follow-up evaluations; and pregnancy or lactation. For each subject, the baseline characteristics, disease duration, clinical presentations and disease severity assessment using the Autoimmune Bullous Skin Disorder Intensity Score (ABSIS) at the start of the combination therapy and throughout subsequent follow-ups were documented. Patients were divided into one of two treatment groups, receiving either AZA or MMF as adjuvant therapy with prednisolone, and treated in parallel. The treatment response, steroid-sparing effect, relapse rate and adverse events of the two groups were compared.

\section{Assessments of Treatment Response}

Treatment response was based on the definitions of disease outcome parameters in the recent international consensus [12] and consisted of of early and late endpoints. The early endpoint assessed in this study was the end of the consolidation phase (ECP), which is defined as the time at which no new lesions have developed for a minimum of 2 weeks and approximately $80 \%$ of lesions have healed. Both the percentages of patients achieving ECP and the median time to ECP were evaluated. The late endpoints were the percentage of patients achieving complete remission (CR) on therapy (defined as the time point when new or established lesions are absent while the patient has been receiving prednisone at $\leq 10 \mathrm{mg} /$ day for at least 2 months), CR off therapy [defined as time point when new or established lesions are absent (ABSIS $=0$ ) while the patient has been off all systemic therapy for at least 2 months] and immunological remission (when positive anti-dsg1 or/and anti-dsg1 has converted to negative values with $20.0 \mathrm{RU}$ (rat units)/mL as 
cutoff value for both antibodies); these were compared in each treatment group.

The median time to achieve each endpoint was also evaluated.

\section{Other Outcome Assessments}

The steroid-sparing effect was assessed using the cumulative steroid dose required to achieve ECP and CR on therapy and CR off therapy in each treatment group. Relapse or flare was defined as the appearance of $\geq 3$ new lesions/month that did not heal spontaneously within 1 week, in a patient who had achieved disease control. All adverse events were also recorded, as well as the rate of drug withdrawal.

\section{Statistical Analysis}

Fisher's exact test or the Chi-squared test was performed for categorical data. The Mann-Whitney test or $t$ test was used on continuous data and the Kaplan-Meier method, log-rank test and Cox regression were used on for time-to-event data. All analyses were conducted on the STATA statistical program version 13 (StataCorp LP, College station, TX, USA). For all tests, a $p<0.05$ was considered to be statistically significant.

\section{RESULTS}

Of the 98 patients with PV or PF included in this study, 28 had received or were receiving multiple immunosuppressants or other treatment modalities, such as, for example, intravenous immunoglobulin, plasmapheresis and/or rituximab, and therefore did not fulfil the inclusion criteria. Eight patients were excluded due to inconsistency regarding the follow-up visits. Of the 62 remaining patients, 37 patients were treated with AZA and 25 patients with MMF as adjuvant therapy with prednisolone. Overall, the baseline characteristics, type of pemphigus, extent of disease, disease duration, follow-up duration, ABSIS and anti-dsg 1/anti-dsg 3 at baseline did not differ between the two groups (Table 1). The initial mean doses of prednisolone in the AZA and MMF groups were also similar (0.53 vs. $0.48 \mathrm{mg} / \mathrm{kg} / \mathrm{day}$, respectively; $p=0.479$ ). In all cases, prednisolone was gradually tapered down according to the individual's clinical response under the management of one expert dermatologist. In the AZA group, patients received AZA at a mean initial dose of $0.83 \mathrm{mg} / \mathrm{kg} / \mathrm{day}$, which was then titrated up to a mean maximum dose of $1.41 \mathrm{mg} /$ $\mathrm{kg} /$ day. In the MMF group, patients received MMF at a mean initial dose of $910 \mathrm{mg} /$ day, which was then titrated up to a mean maximum dose of $1530 \mathrm{mg} /$ day.

A majority of patients in both groups (AZA group 88.2\%; MMF group 71.4\%) reached the early endpoint (ECP), with no significant between-group difference ( $p=0.156$ ) (Table 2); the median time required to achieve this early endpoint was also comparable [AZA: 2.9 months, 95\% confidence interval (CI) 2.1, 4.4; MMF: 3.5 months, 95\% CI 1.6, 5.9; $p=0.362$ ) (Fig. 1).

CR when on therapy was observed in 73 and $72 \%$ of patients receiving AZA and MMF as adjuvant, respectively $(p=0.933)$ (Table 2$)$. However, the median time to achieve CR on therapy was significantly shorter in the MMF group than in the AZA group (7.3 vs. 12.5 months; $p=0.019$ ) (Fig. 2). The hazard ratio for CR on therapy for the MMF group compared to the AZA group was 2.07 (95\% CI 1.11, 3.86) within the follow-up period ( $p=0.021$; Table 3 ). After adjusting for all relevant confounding factors, including baseline ABSIS, age, diagnosis (PV, PF), type of patient (new, partially treated, relapsed), extent of involvement and presence of complications, the hazard ratio for CR on therapy for the MMF group compared to the AZA group was $2.55(95 \%$ CI 1.15, 5.63) and remained significant $(p=0.021)$. Subgroup analysis showed that patients with PV, but not those with $\mathrm{PF}$, who received MMF had a shorter time to achieve CR on therapy than did patients receiving AZA (6.5 vs. 14.4 months), but this difference did not reach statistical significance $(p=0.093)$. For CR off therapy, the rates were 24.3 and $20 \%$ in AZA group and MMF group, respectively $(p=0.690)$ (Table 2). The median time to achieve this endpoint appeared to be shorter for the MMF group than for the AZA group (29.5 vs. 52.3 months), but the difference was not 
Table 1 Baseline characteristics of the treatment groups

\begin{tabular}{|c|c|c|c|}
\hline Characteristics & $\begin{array}{l}\text { AZA group } \\
(n=37)\end{array}$ & $\begin{array}{l}\text { MMF group } \\
(n=25)\end{array}$ & $p$ value \\
\hline Sex (male/female), $n$ & $16 / 21$ & $7 / 18$ & 0.223 \\
\hline Diagnosis $(\mathrm{PV} / \mathrm{PF}), n$ & $29 / 8$ & $15 / 10$ & 0.118 \\
\hline $\begin{array}{l}\text { Extent of involvement (mucosal only/cutaneous } \\
\text { only/mucocutaneous), } n\end{array}$ & $6 / 8 / 23$ & $4 / 12 / 9$ & 0.074 \\
\hline Patient category (new/old/relapse), $n$ & $23 / 9 / 5$ & $12 / 11 / 2$ & 0.256 \\
\hline Age at onset (years), mean (SD) & $44.54(13.68)$ & $55.13(17.36)$ & 0.062 \\
\hline Age at diagnosis (years), mean (SD) & $44.92(13.78)$ & $52.29(17.30)$ & 0.070 \\
\hline Age at start adjuvant (years), mean (SD) & $46.27(13.71)$ & $52.4(17.22)$ & 0.125 \\
\hline Disease duration (months), median (range) & $10(0.5180)$ & $6(0.5,96)$ & 0.959 \\
\hline Follow-up duration (days), median (range) & $595(7,2363)$ & $399(13,1834)$ & 0.199 \\
\hline Baseline ABSIS, median (range) & $4.5(0.25,43.75)$ & $4(1.125,41)$ & 0.293 \\
\hline Baseline anti-Dsg-1 $(\leq 20 / 21-100 / 101-200 />200 \mathrm{U} / \mathrm{mL}), n$ & $1 / 6 / 7 / 15$ & $2 / 5 / 3 / 14$ & 0.677 \\
\hline Baseline anti-Dsg-3 ( $\leq 20 / 21-100 / 101-200 />200 \mathrm{U} / \mathrm{mL}), n$ & $5 / 3 / 5 / 16$ & $8 / 4 / 6 / 5$ & 0.101 \\
\hline Baseline prednisolone $(\mathrm{mg} / \mathrm{kg})$, mean $(\mathrm{SD})$ & $0.53(0.24)$ & $0.48(0.21)$ & 0.479 \\
\hline
\end{tabular}

ABSIS Autoimmune Bullous Skin Disorder Intensity Score, AZA Azathioprine, $D s g-1$ desmoglein-1, $D s g-3$ desmoglein-3, $n$ number of patients, $M M F$ mycophenolate mofetil, $P F$ pemphigus foliaceus, $P V$ pemphigus vulgaris, $S D$ standard deviation

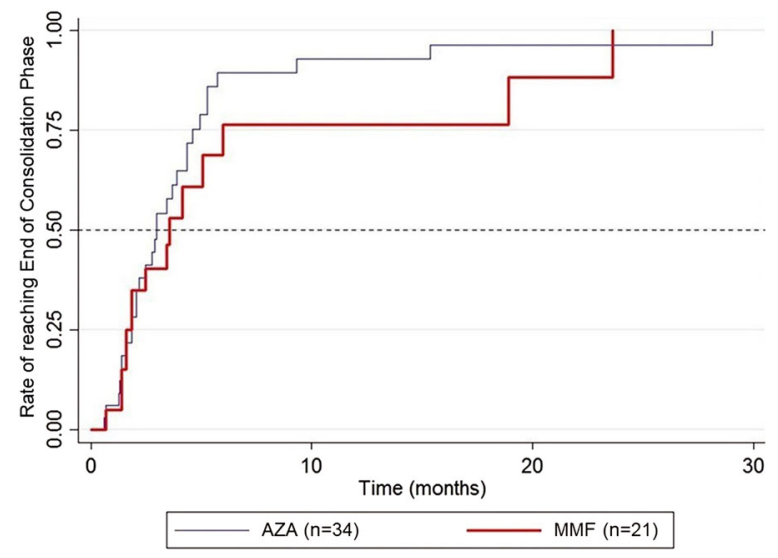

Fig. 1 Kaplan-Meier plot showing the rate of patients reaching the early endpoint (end of the consolidation phase) for each treatment. $A Z A$ Azathioprine, $M M F$ mycophenolate mofetil

statistically significant $(p=0.110) \quad$ (Fig. 3). Immunological remission was detected in approximately one-third of patients in each group, with no significant between-group

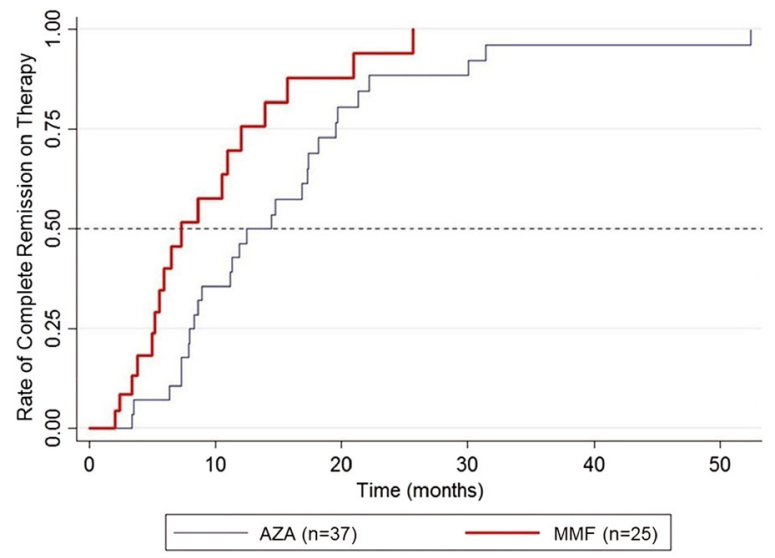

Fig. 2 Kaplan-Meier plot showing the rate of complete remission in patients on therapy for each treatment

difference $(p=0.913)$. Although the median time to immunological remission was shorter in the MMF versus the AZA group (15.3 vs. 41.9 months), this difference did not reach statistical significance $(p=0.157)$ (Fig. 4). 
Table 2 Treatment response and cumulative steroid use in each group

\begin{tabular}{llll}
\hline Outcomes & AZA $(\boldsymbol{n}=37)$ & MMF $(\boldsymbol{n}=25)$ & $\boldsymbol{p}$ value \\
\hline No. of patients reaching ECP & & 0.156 \\
PV + PF & $30 / 34(88.24 \%)$ & $15 / 21(71.43 \%)$ & 0.221 \\
PV & $23 / 27(85.19 \%)$ & $8 / 12(66.67 \%)$ & 0.475 \\
PF & $7 / 7(100 \%)$ & $7 / 9(77.78 \%)$ & 0
\end{tabular}

No. of patients achieving CR on therapy

$$
\begin{array}{ll}
\mathrm{PV}+\mathrm{PF} & 27 / 37(72.97 \%) \\
\mathrm{PV} & 22 / 29(75.86 \%) \\
\mathrm{PF} & 5 / 8(62.50 \%)
\end{array}
$$

$$
18 / 25(72 \%)
$$

$11 / 15(73.33 \%)$

$7 / 10(70 \%)$

No. of patients achieving CR off therapy

$$
\begin{array}{ll}
\mathrm{PV}+\mathrm{PF} & 9 / 37(24.32 \%) \\
\mathrm{PV} & 7 / 29(24.14 \%) \\
\mathrm{PF} & 2 / 8(25 \%)
\end{array}
$$$$
5 / 25(20 \%)
$$$$
0.69
$$$$
3 / 15(20 \%)
$$$$
1.00
$$

$2 / 10(20 \%)$

1.00

No. of patients achieving immunological remission

$$
\begin{array}{ll}
\mathrm{PV}+\mathrm{PF} & 13 / 36(36.11 \%) \\
\mathrm{PV} & 12 / 28(42.86 \%) \\
\mathrm{PF} & 1 / 8(12.50 \%)
\end{array}
$$

$9 / 24(37.50 \%)$

0.913

$5 / 14(35.71 \%)$ 0.657

Median time (months) to reach ECP (95\% CI)

$$
\begin{array}{ll}
\mathrm{PV}+\mathrm{PF} & 2.9(2.1,4.4) \\
\mathrm{PV} & 3.7(2.1,4.6) \\
\mathrm{PF} & 1.8(0.6,2.9)
\end{array}
$$$$
4 / 10(40 \%)
$$

$3.5(1.6,5.9)$

0.362

$4.1(1.8,-)$

0.319

$1.6(1.4,-)$

0.329

Median time (months) to achieve CR on therapy (95\% CI)

$$
\begin{array}{ll}
\mathrm{PV}+\mathrm{PF} & 12.5(8.7,17.4) \\
\mathrm{PV} & 14.4(8.9,18.2) \\
\mathrm{PF} & 8.3(3.5,-)
\end{array}
$$$$
7.3(5.2,12.3)
$$$$
0.019^{*}
$$$$
6.5(3.8,15.7)
$$$$
0.093
$$$$
8.6(3.4,10.9)
$$

Median time (months) to achieve CR off therapy (95\% CI)

$$
\begin{array}{ll}
\mathrm{PV}+\mathrm{PF} & 52.3(49.7,60.5) \\
\mathrm{PV} & 52.2(49.7,-) \\
\mathrm{PF} & 55.0(33.4,-)
\end{array}
$$$$
29.5(17.8,-)
$$$$
0.11
$$$$
29.5(18,-)
$$$$
0.370
$$$$
17.8(14.4,-)
$$

Median time (months) to achieve immunological remission (95\% CI)

\begin{tabular}{llll}
$\mathrm{PV}+\mathrm{PF}$ & $41.9(15.0,-)$ & $15.3(5.38,-)$ & 0.157 \\
$\mathrm{PV}$ & $41.4(13.3,-)$ & $15.6(11.7,-)$ & 0.527 \\
$\mathrm{PF}$ & $-(4.2,-)$ & $5.4(4.9,-)$ & 0.255 \\
\hline
\end{tabular}


Table 2 continued

\begin{tabular}{llll}
\hline Outcomes & AZA $(\boldsymbol{n}=\mathbf{3 7})$ & MMF $(\boldsymbol{n}=\mathbf{2 5})$ & $\boldsymbol{p}$ value \\
\hline $\begin{array}{l}\text { Cumulative steroid level at ECP }(\mathrm{mg}) \text {, median (range) } \\
\text { PV + PF }\end{array}$ & $2730(360,20,175)$ & $1480(700,12,127.5)$ & 0.63 \\
PV & $2905(420,20,175)$ & $2022.5(840,12,127.5)$ & 0.588 \\
PF & $1680(360,4550)$ & $1330(700,5985)$ & 0.749 \\
Cumulative steroid level at CR on therapy (mg), median (range) & & \\
PV + PF & $7740(610,42,990)$ & $3473.8(397.5,12,367.5)$ & $0.007^{*}$ \\
PV & $8583.75(610,42,990)$ & $3252.5(397.5,12,367.5)$ & $0.012^{*}$ \\
PF & $4080(1342.5,12670)$ & $4117.5(1262.5,9010)$ & 0.569 \\
Cumulative steroid level at CR off therapy (mg), mean (SD) & & \\
PV + PF & $12,857.3(4491.71)$ & $7590.5(3428.34)$ & $0.043^{*}$ \\
PV & $11621.11(4065.65)$ & $8380.83(3937.04)$ & 0.278 \\
PF & $17,185(3751.20)$ & $6405(3365.82)$ & 0.094 \\
\hline
\end{tabular}

ECP End of the consolidation phase (early endpoint)

${ }^{*} p$ value $<0.05$, indicating a statistically significant difference

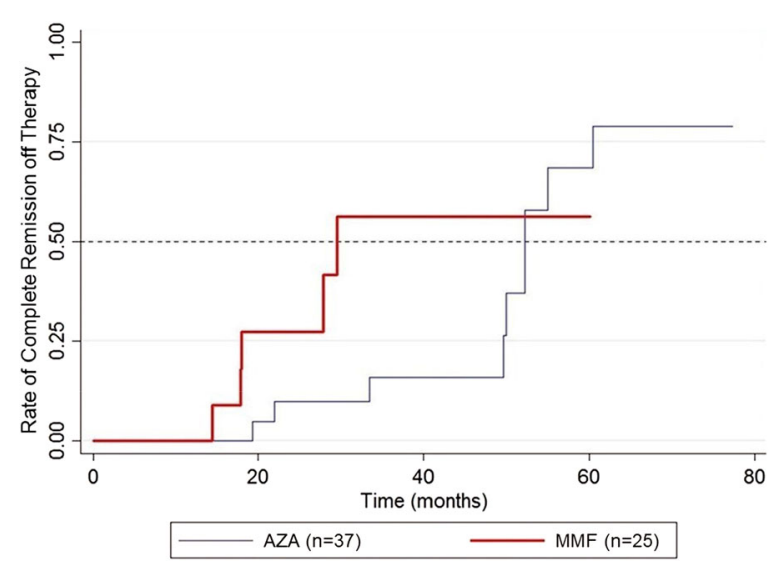

Fig. 3 Kaplan-Meier plot showing the rate of complete remission in patients off therapy for each treatment

\section{Steroid-Sparing Effect, Pemphigus Recurrence and Adverse Events}

The cumulative dosage of prednisolone required to achieve ECP was lower in the MMF group than in the AZA group, but the difference did not reach statistical significance $(p=0.630)$. However, the cumulative doses required for

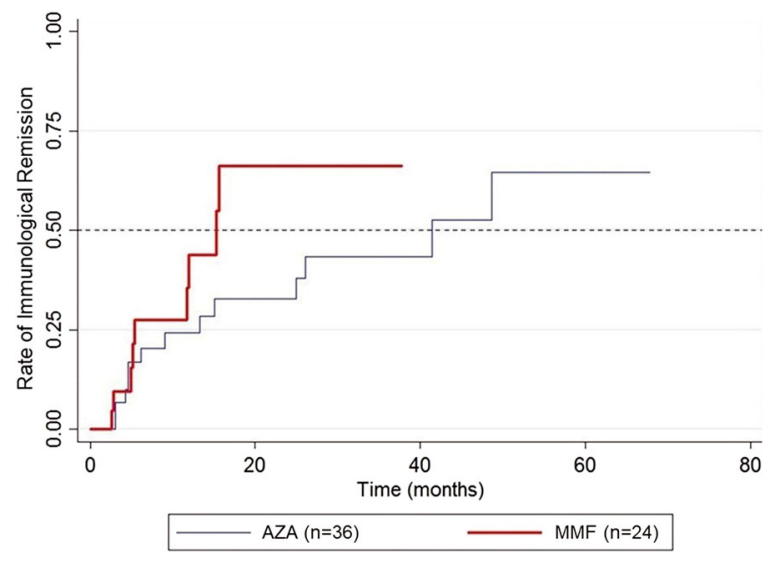

Fig. 4 Kaplan-Meier plot showing the rate of immunological remission in patients for each treatment

patients to achieve CR both on and off therapy were significantly lower in the MMF group ( $p=0.007$ and $p=0.043$, respectively), indicating that MMF has a more superior steroid-sparing effect than AZA. Subgroup analysis on PV patients also showed a statistically lower cumulative dose of prednisolone at $\mathrm{CR}$ on therapy in the MMF group ( $p=0.012)$ (Table 2). 
Table 3 Hazard ratio and 95\% confidence interval of the treatment effect on complete remission in patients when on therapy generated by the Cox proportional hazard model

\begin{tabular}{lllll}
\hline Characteristic & $\begin{array}{l}\text { Crude HR }(95 \% \mathrm{CI}) \text { for } \\
\text { CR when on therapy }\end{array}$ & $\boldsymbol{p}$ value & $\begin{array}{l}\text { Adjusted } \mathrm{HR}^{\mathbf{a}}(\mathbf{9 5 \%} \mathrm{CI}) \\
\text { for CR when on therapy }\end{array}$ & $\boldsymbol{p}$ value \\
\hline $\begin{array}{l}\text { Treatment } \\
\text { MMF }\end{array}$ & $2.07(1.11-3.86)$ & 0.021 & $2.55(1.15-5.63)$ & 0.021 \\
AZA & 1 & & 1 & \\
\hline
\end{tabular}

$A Z A$ azathioprine, $C I$ confidence interval, $C R$ complete remission, $H R$ hazard ratio, $M M F$ mycophenolate mofetil

a Adjusted forbaseline ABSIS, age, diagnosis (PV, PF), type of patient (new, partially treated, relapsed), extent of involvement and presence of complication

Recurrence occurred in a small proportion of patients in both groups. Of 30 patients who achieved ECP in the AZA group, four experienced recurrence (after 8, 23, 226 and 305 days, respectively), while one of $15 \mathrm{MMF}$ patients achieving ECP had recurrence (after 58 days). Due to the small number of recurrences, the statistical analysis for the between-group comparison was not performed. Table 4 summarizes the adverse events. No statistically significant differences in the type and rate of each adverse event and in the incidence of drug discontinuation due to the adverse events were observed (21.6 and $12 \%$ in the AZA and MMF groups, respectively; $p=0.330$ ). No death was reported in either group.

\section{DISCUSSION}

The chronic autoimmune nature of pemphigus requires longstanding treatment with corticosteroids, which often leads to severe adverse events [3]. Combination therapy with various adjuvants has long been a subject of interest and consistently included in previous national and international treatment guidelines [8-12]. AZA (1-3 mg/kg/day) and MMF (30-45 mg/ $\mathrm{kg} /$ day) are both listed as "first line adjuvants" in the European international guideline [11] and as "first line corticosteroid sparing agents" in the recent international expert panel recommendations [12].

Numerous studies have compared corticosteroid with either AZA or MMF as adjuvant (combination therapy) with corticosteroid monotherapy, but only a few studies have directly compared these two combination therapeutic modalities [23-25]. In terms of treatment response, our results suggest that while patients receiving corticosteroid in combination with AZA or MMF achieved similarly high rates of remission, MMF was associated with better outcomes in terms of a significantly shorter time to CR on therapy and a trend towards shorter time to CR off therapy. These results are contradictory to those reported in a previous study in which patients on AZA were shown to have a significantly shorter mean duration to remission (defined as complete reepithelization of all previous lesions) [23]. This difference may be explained by the difference in the outcome parameters. The endpoints used in this earlier study may actually be more comparable to the time required to achieve the ECP in our study; we also found this endpoint (defined as an early endpoint in our study) to be shorter in the AZA group than in the MMF group, but the difference was not statistically significant. The shorter time to CR in the patients receiving MMF in our study also translated into a significantly lower cumulative steroid dose in this group. This result highlights in particular the superiority of MMF over AZA as these adjuvant immunosuppressants were prescribed primarily to reduce the use of corticosteroid. In contrast to our findings, Chams-Davatchi et al. reported a lower mean total dose of prednisolone administered in the AZA group when compared to the MMF group during their treatment period of 1 year [24]. However, in their study the cumulative corticosteroid dose was calculated after a fixed period of 1 year, whereas in our 
Table 4 Summary of adverse events in pemphigus patients receiving azathioprine or mycophenolate mofetil as adjuvant therapy with prednisolone

\begin{tabular}{|c|c|c|c|}
\hline Adverse events & $\operatorname{AZA}(n=37)$ & MMF $(n=25)$ & $p$ value \\
\hline Oral candidiasis & $7(18.92 \%)$ & $2(8 \%)$ & 0.29 \\
\hline Dermatophyte infection & $2(5.41 \%)$ & $0(0 \%)$ & 1 \\
\hline Cellulitis & $2(5.41 \%)$ & $3(12 \%)$ & 0.39 \\
\hline Pneumonia & $1(2.7 \%)$ & $1(4 \%)$ & 1 \\
\hline Hyperglycemia & $6(4.2 \%)$ & $1(4 \%)$ & 0.23 \\
\hline Fever & $5(2.41 \%)$ & $0(0 \%)$ & 0.51 \\
\hline Abdominal discomfort & $1(2.7 \%)$ & $0(0 \%)$ & 1 \\
\hline Hepatitis & $4(10.81 \%)$ & $1(4 \%)$ & 0.64 \\
\hline Meningitis & $1(2.7 \%)$ & $0(0 \%)$ & 1 \\
\hline TB infection & $1(2.7 \%)$ & $0(0 \%)$ & 1 \\
\hline Nausea/vomiting & $1(2.7 \%)$ & $0(0 \%)$ & 1 \\
\hline Diarrhea & $0(0 \%)$ & $1(4 \%)$ & 0.16 \\
\hline Herpes zoster & $2(5.41 \%)$ & $2(8 \%)$ & 1 \\
\hline URI & $0(0 \%)$ & $2(8 \%)$ & 0.16 \\
\hline Mild transaminitis & $2(5.41 \%)$ & $0(0 \%)$ & 0.51 \\
\hline $\begin{array}{l}\text { Bone marrow } \\
\text { suppression }\end{array}$ & $2(5.41 \%)$ & $0(0 \%)$ & 1 \\
\hline Eczema herpeticum & $0(0 \%)$ & $1(4 \%)$ & 0.4 \\
\hline $\begin{array}{l}\text { Adverse events leading } \\
\text { to drug withdrawal }\end{array}$ & $\begin{array}{l}8 \text { (21.6\%): hepatitis (4), meningitis (1), TB } \\
\text { infection (1), BM suppression (2) }\end{array}$ & $\begin{array}{l}3 \text { (12\%): hepatitis (1), pneumonia (1), } \\
\text { cellulitis followed by DVT (1) }\end{array}$ & 0.33 \\
\hline
\end{tabular}

Values in table are presented as the number of patients with the respective percentage of the treatment group given in parenthesis

$B M$ Bone marrow, $D V T$ deep vein thrombosis, $T B$ tuberculosis, $U R I$ upper respiratory tract infection

study the total cumulative dose was calculated up to the time of CR.

Our results appear to indicate that the recurrence rate was higher in the AZA group, but the statistical significance of the difference could not be determined due to the small number of recurrences and the difference in number of patients achieving the ECP in each group. In two previous studies, the rate of and time to recurrence were similar in patients receiving AZA or MMF [23, 25]. Our results show that the rate and type of adverse event did not differ between the two treatment groups, a finding consistent with results reported previously [23, 24]. Like earlier studies, the majority of adverse events were minor, with candidiasis being the most common [24], and findings of elevated liver enzymes were also prevalent, with a slightly higher proportion observed in the AZA group [23].

There are a few limitations to our study. Due to the retrospective design, some data in the electronic records may have been incomplete and the follow-up intervals may not be uniform in all patients. Although the patients were treated in the same clinic by the same expert 
dermatologist, tapering of both corticosteroid and AZA/MMF may have varied between individuals. However, our data reflect real-life scenarios and demonstrate the performance of each adjuvant when given in clinical practice. In the future, findings from prospective controlled trials with standardized endpoints may support these results.

\section{CONCLUSION}

The results of this study suggest that corticosteroid in combination with either AZA or MMF is an effective treatment regimen for pemphigus, based on similarly high rates of patients in either treatment group achieving remission. However, MMF was associated with a significantly shorter time to achieve CR on therapy and a significantly higher steroid-sparing effect, indicating a higher potential for lowering the risk of adverse events associated with prolonged steroid use. Further prospective controlled trials with standardized disease outcomes are required to confirm this superiority over AZA.

\section{ACKNOWLEDGEMENTS}

Funding. This is an investigator-initiated study, and no funding or sponsorship was received for this study or publication of this article. The journal's Rapid Service Fee was funded by the authors.

Authorship. All named authors meet the International Committee of Medical Journal Editors (ICMJE) criteria for authorship for this article, take responsibility for the integrity of the work as a whole, and have given their approval for this version to be published.

Disclosures. Siriorn Sukanjanapong, Darin Thongtan, Silada Kanokrungsee, Poonkiat Suchonwanit and Kumutnart Chanprapaph have nothing to disclose.

Compliance with Ethics Guidelines. This retrospective analytic study was approved by the Mahidol University Institutional Review Board (ID 08-60-74). All procedures performed in studies involving human participants were in accordance with the institutional research committee and with the 1964 Helsinki Declaration and its later amendments or comparable ethical standards. Informed consent was obtained from all individual participants included in the study.

Data Availability. The datasets during and/ or analyzed during the current study are available from the corresponding author on reasonable request.

Open Access. This article is licensed under a Creative Commons Attribution-NonCommercial 4.0 International License, which permits any non-commercial use, sharing, adaptation, distribution and reproduction in any medium or format, as long as you give appropriate credit to the original author(s) and the source, provide a link to the Creative Commons licence, and indicate if changes were made. The images or other third party material in this article are included in the article's Creative Commons licence, unless indicated otherwise in a credit line to the material. If material is not included in the article's Creative Commons licence and your intended use is not permitted by statutory regulation or exceeds the permitted use, you will need to obtain permission directly from the copyright holder. To view a copy of this licence, visit http://creativecommons.org/licenses/bync/4.0/.

\section{REFERENCES}

1. Chams-Davatchi C, Valikhani M, Daneshpazhooh M, et al. Pemphigus: analysis of 1209 cases. Int J Dermatol. 2005;44(6):470-6.

2. Pisanti S, Sharav Y, Kaufman E, Posner LN. Pemphigus vulgaris: incidence in Jews of different ethnic groups, according to age, sex, and initial lesion. Oral Surg Oral Med Oral Pathol. 1974;38(3):382-7.

3. Bystryn JC, Steinman NM. The adjuvant therapy of pemphigus. An update. Arch Dermatol. 1996;132(2):203-12. 
4. Atzmony L, Hodak E, Gdalevich M, Rosenbaum O, Mimouni D. Treatment of pemphigus vulgaris and pemphigus foliaceus: a systematic review and metaanalysis. Am J Clin Dermatol. 2014;15(6):503-15.

5. Atzmony L, Hodak E, Leshem YA, et al. The role of adjuvant therapy in pemphigus: a systematic review and meta-analysis. J Am Acad Dermatol. 2015;73(2):264-71.

6. Martin LK, Werth VP, Villaneuva EV, Murrell DF. A systematic review of randomized controlled trials for pemphigus vulgaris and pemphigus foliaceus. J Am Acad Dermatol. 2011;64(5):903-8.

7. Mimouni D, Nousari CH, Cummins DL, Kouba DJ, David M, Anhalt GJ. Differences and similarities among expert opinions on the diagnosis and treatment of pemphigus vulgaris. J Am Acad Dermatol. 2003;49(6):1059-62.

8. Committee for Guidelines for the Management of Pemphigus Disease, Amagai M, Tanikawa A, et al. Japanese guidelines for the management of pemphigus. J Dermatol. 2014;41(6):471-86.

9. Eming R, Sticherling M, Hofmann SC, et al. S2k guidelines for the treatment of pemphigus vulgaris/foliaceus and bullous pemphigoid. J Dtsch Dermatol Ges. 2015;13(8):833-44.

10. Harman KE, Brown D, Exton LS, et al. British Association of Dermatologists' guidelines for the management of pemphigus vulgaris 2017. Br J Dermatol. 2017;177(5):1170-201.

11. Hertl M, Jedlickova H, Karpati S, et al. Pemphigus. S2 Guideline for diagnosis and treatment-guided by the European Dermatology Forum (EDF) in cooperation with the European Academy of Dermatology and Venereology (EADV). J Eur Acad Dermatol Venereol. 2015;29(3):405-14.

12. Murrell DF, Pena S, Joly $\mathrm{P}$, et al. Diagnosis and management of pemphigus: recommendations by an International Panel of Experts. J Am Acad Dermatol. 2018. https://doi.org/10.1016/j.jaad.2018. 02.021 .

13. Murrell DF, Dick S, Ahmed AR, et al. Consensus statement on definitions of disease, end points, and therapeutic response for pemphigus. J Am Acad Dermatol. 2008;58(6):1043-6.

14. Kazlow Stern D, Tripp JM, Ho VC, Lebwohl M. The use of systemic immune moderators in dermatology: an update. Dermatol Clin. 2005;23(2): 259-300.
15. Chams-Davatchi C, Mortazavizadeh A, Daneshpazhooh M, et al. Randomized double blind trial of prednisolone and azathioprine, vs. prednisolone and placebo, in the treatment of pemphigus vulgaris. J Eur Acad Dermatol Venereol. 2013;27(10): 1285-92.

16. Aberer W, Wolff-Schreiner EC, Stingl G, Wolff K. Azathioprine in the treatment of pemphigus vulgaris. A long-term follow-up. J Am Acad Dermatol. 1987;16(3 Pt 1):527-33.

17. Orvis AK, Wesson SK, Breza TS Jr, Church AA, Mitchell CL, Watkins SW. Mycophenolate mofetil in dermatology. J Am Acad Dermatol. 2009;60(2): 183-99 (quiz 200-202).

18. Enk AH, Knop J. Treatment of pemphigus vulgaris with mycophenolate mofetil. Lancet. 1997; 350(9076):494.

19. Enk AH, Knop J. Mycophenolate is effective in the treatment of pemphigus vulgaris. Arch Dermatol. 1999;135(1):54-6.

20. Chams-Davatchi C, Nonahal Azar R, Daneshpazooh $\mathrm{M}$, et al. Open trial of mycophenolate mofetil in the treatment of resistant pemphigus vulgaris. Ann Dermatol Venereol. 2002;129(1 Pt 1):23-5.

21. Beissert S, Mimouni D, Kanwar AJ, Solomons N, Kalia V, Anhalt GJ. Treating pemphigus vulgaris with prednisone and mycophenolate mofetil: a multicenter, randomized, placebo-controlled trial. J Invest Dermatol. 2010;130(8):2041-8.

22. Ioannides D, Apalla Z, Lazaridou E, Rigopoulos D. Evaluation of mycophenolate mofetil as a steroidsparing agent in pemphigus: a randomized, prospective study. J Eur Acad Dermatol Venereol. 2012;26(7):855-60.

23. Beissert $\mathrm{S}$, Werfel T, Frieling U, et al. A comparison of oral methylprednisolone plus azathioprine or mycophenolate mofetil for the treatment of pemphigus. Arch Dermatol. 2006;142(11):1447-54.

24. Chams-Davatchi C, Esmaili N, Daneshpazhooh M, et al. Randomized controlled open-label trial of four treatment regimens for pemphigus vulgaris. J Am Acad Dermatol. 2007;57(4):622-8.

25. Esmaili N, Chams-Davatchi C, Valikhani M, et al. Assessment of the therapeutic benefit of oral prednisolone and common adjuvant therapy in stage II of randomized controlled trial study for management of pemphigus vulgaris. Arch Iran Med. 2014;17(9):626-8. 